Pacific Journal of Mathematics

COVERING GROUPS OF GROUPS OF LIE TYPE 


\title{
COVERING GROUPS OF GROUPS OF LIE TYPE
}

\section{JOHN GROVER}

\begin{abstract}
A construction for a central extension of a group satisfying a certain set of axioms has been given by $C$. W. Curtis. These groups are called groups of Lie type. The construction is based on that given by $R$. Steinberg for covering groups of the Chevalley groups. The central extensions constructed by Curtis, however, are not covering groups in the sense of being universal central extensions, as he shows by an example. Here, the Steinberg construction is considered for a more restricted class of groups of Lie type. It is shown that in this case, the central extension is a covering. It is also shown that this more restricted definition of groups of Lie type still includes the Chevalley and twisted groups, with certain exceptions.
\end{abstract}

To fix our terminology: a universal central extension is one which factors through any other central extension. A covering is a universal central extension, no subgroup of which is also an extension of the same group. $(x, y)=x y x^{-1} y^{-1}, a^{b}=b a b^{-1}$, and $(G, G)$ is the commutator subgroup of $G . G$ is perfect if $G=(G, G) . L_{n}(K)$ denotes the Chevalley group of type $L$ and rank $n$ over the field $K$. Twisted groups are defined here to be the (algebraic) nonnormal forms as constructed by D. Hertzig [5, 6], R. Steinberg [9] and J. Tits $[14,15]$. (Hertzig also shows that the Chevalley and twisted groups include all finite simple algebraic groups.)

The Chevalley groups are simple $[1,10,16]$, hence perfect. This means that a perfect covering group exists, and the covering group and its factor maps are unique. Steinberg shows that the covering can be constructed as the abstract group given by suitably chosen generators and relations from the Chevalley group [11]. Exceptions occur: $A_{1}(2), A_{1}(3), B_{2}(2)$ and $G_{2}(2)$ are not simple, and Steinberg's construction doesn't work when $|K|=2$, 3, or 4 , or $G=A_{1}(9)$.

The construction of the covering group $\Delta$ easily extends to a group $G$ with Bruhat decomposition as defined by Curtis [2]. However, in this case $\Delta$ need not be the covering group of $G$. By placing additional conditions on $G$, Curtis shows that $\Delta$ has a Bruhat decomposition, with the same (isomorphic) Weyl group as $G$, is a central extension, and is "almost" universal [3, Ths. 1.4, 1.7].

The next section gives the set of axioms which characterize the class of groups of Lie type. Our main theorem (6.2) can be stated as: 
The Steinberg group of a group of Lie type is its covering group.

Some other results are derived on various types of unique expression in $G$ and in $\Delta$, and on the structure of the subgroups $N$ and $H$. The results apply to most of the Chevalley groups and twisted groups. The last section specifies exactly which groups. In what follows, $G$ is understood to have rank not one. The rank one case is discussed separately in the next-to-last section.

2. Definition of groups of Lie type. The notation is fixed from now on. $G$ is an arbitrary group of Lie type, that is, an arbitrary group satisfying conditions (2.1) through (2.11) below.

(2.1) $G$ has subgroups $B, N$ such that $H=B \cap N$ is normal in $N, B=U H$ for some subgroup $U$ normal in $B$, and $U \cap H=1$.

(2.2) $W=N / H$ is (isomorphic to) a crystallographic reflection group.

Let $t: W \rightarrow N$ be a fixed inverse map to the natural homomorphism $s: N \rightarrow W$. Sometimes elements of $W$ will be used in place of their images under $t$ without ambiguity as in $U^{w}$.

(2.3) $U^{w_{0}} \cap B=1$ for some $w_{0} \in W$.

Choose fixed fundamental generators $\left\{w_{1}, w_{2}, \cdots, w_{n}\right\}$ and a fixed $w_{0}$ in $W$ by (2.2) and (2.3). Define $U_{w}=\left\{u \in U: u^{w} \in U\right\}$ and $R_{i}=$ $U_{w_{0} w_{i}}$ for $w \in W$ and $i=1,2, \cdots, n$.

(2.4) $R_{i} \neq 1$ and $R_{i} \subseteq U_{w}$ or $R_{i} \subseteq U_{w w_{i}}$ all $w, i$.

(2.5) $G_{i}=\left(R_{i} H\right) \cup\left(R_{i} H w_{i} R_{i}\right)$ is a subgroup, $i=1,2, \cdots n$.

(2.6) $G=\langle\Sigma\rangle$ and $U=\langle P\rangle$.

Here $\Sigma=\left\{R_{i}^{n}: n \in N, i=1,2, \cdots, n\right\}, \quad P=\{R \in \Sigma: R \cong U\}$, and $\langle x y \cdots\rangle$ means the subgroup generated by $x y \cdots H$ normalizes each $R \in \Sigma$, so elements of $W$ act on $\Sigma$ by conjugation by their images by $t$. This action agrees with the action of $W$ on its roots, and in fact $\Sigma$ may be identified with the roots of $W$ as in [2, Prop. 3.2]. Hence reflections $w_{R}$, negatives $-R$, and (sometimes) sums $R+S$ are defined in $\Sigma$.

(2.7) Given $R \in \Sigma, y \in-R \backslash\{1\}$, there exists $x \in R$ such that $x y x \in N$ and $s(x y x)=w_{R}$.

(2.8) Given $R, S \in \Sigma, R \neq-S$ and $r \in R, s \in S$, there exist $\mathrm{t}_{\imath j} \in i R+j S$ such that $(r, s)=\pi t_{i j}$. The product is over roots of the form $i R+j S$, $i, j$ positive integers. Every $t$ in each $T \in \Sigma$ appears as a $t_{i j}$ in some relation of this form.

Every element of $\Sigma$ is a linear combination of fundamental roots $R_{i}$. Define the lexicographical ordering of $\Sigma$ by $R=\Sigma a_{\imath} R_{i}>S=\Sigma b_{i} R_{i}$ if $a_{i}>b_{i}$ for the first $i$ such that $a_{i} \neq b_{i}$.

(2.9) $R \cap\langle S: S \in \Sigma \& S>R\rangle=1$ for all $R \in P$. 
(2.10) For $R \in \Sigma$, there exists $h \in H$ such that the map $x \rightarrow(h, x)$ : $R \rightarrow R$ is one-to-one and onto.

(2.11) For $R, S \in \Sigma, R \neq \pm S$, there exists $h \in H$ such that $(h, R)=1 \&(h, S)=S$, or $(h, R)=R \&(h, S)=1$.

3. Structure of groups of Lie type. Since $G$ satisfies additional conditions besides those imposed on a group with Bruhat decomposition, its structure is more precisely determined. Some of this additional structure is developed here, being needed in what follows.

Proposition 3.1. Every element of $U$ has a unique expression as a product of elements of distinct subgroups $R \in P$, arranged in ascending order.

By (2.6), $U=\langle P\rangle$, so every $x \in U$ has an expression as a product of such elements. Condition (2.8) and induction on root order allow such a product to be arranged by ascending root order. Finally, (2.9) implies the uniqueness of the rearranged product.

The same proof actually shows unique expression holds in any "convex" subgroup. A subgroup is convex if it is generated by a convex set of root subgroups. A set of roots is convex if it is additively closed and positive relative to some ordering of $\Sigma$. A splitting of $P$ is a partition into two additively closed root sets, i.e., a convex partition.

Lemma 3.2. If $P^{\prime}, P^{\prime \prime}$ split $P$, then $P=\left\langle P^{\prime}\right\rangle\left\langle P^{\prime \prime}\right\rangle$.

As in the proof of (3.1), (2.8) is used to rearrange out-of-place factors, and the lemma follows by induction on root order. If $R_{i}$ is a fundamental root for example, $\left\{R_{i}\right\}$ and $P \backslash\left\{R_{i}\right\}$ are a splitting of $P$, so $P=\left\langle R_{\imath}\right\rangle\left\langle P \backslash\left\{R_{i}\right\}\right\rangle=R_{i} U_{w_{i}}$.

Proposition 3.3. G has a Bruhat decomposition.

Conditions (c), (d), (g), (h) of Curtis' definition [2] correspond to conditions (2.2), (2.3), (2.5) and (2.6) respectively. (2.1) implies (a) and (b), (2.4) implies (f), and with the remark following (3.2), also implies (e).

All Curtis' results on groups with a Bruhat decomposition apply to $G$ : $B$ and $N$ form a $B N$-pair for $G, B w B=B w^{\prime} B$ implies $w=w^{\prime}$, and $G=B N B$. The additional structure of $G$ implies that the decomposition $G=\Sigma_{W} U H w U_{w_{0} w}$ is unique.

Proposition 3.4. The form of this decomposition, for elements 
of $G$, is unique.

Suppose $u h t(w) v=u^{\prime} h^{\prime} t\left(w^{\prime}\right) v^{\prime}$. Then $w=w^{\prime}$, and $h^{-1} u^{-1} u^{\prime} h^{\prime}=$ $\left(v v^{\prime-1}\right)^{t(w)}$. But $v, v^{\prime} \in U_{w_{0} w}$, so $\left(v v^{\prime-1}\right)^{t(w)} \in U^{w_{0}}=V$, and $B \cap V=1$ by (2.3). Hence $v=v^{\prime}$, and then $h h^{\prime-1}=u^{-1} u^{\prime} \in H \cap U=1$, so $h=h^{\prime}$ and $u=u^{\prime}$. Thus the elements in the Bruhat decomposition are unique, up to a fixed choice of $t$.

An element $x y x$ of the form given by (2.7) is denoted by $n(R, x, y)$. It will be shown these generate $N$ and $H$.

Lемма 3.5. A unique element $n(R, x, y)$ exists for each $x \in R \backslash\{1\}$, and for each $y \in-R \backslash\{1\}$.

Choose $\mathrm{y} \in-R \backslash\{1\}$. Then $n(R, x, y)$ exists by (2.7). Also, $y=y^{\prime}$ is implied by $n(R, x, y)=n\left(R, x, y^{\prime}\right)$. The existence of $y$ given $x$, and the uniqueness of $x$ given $y$, follow the identity $n(R, x, y)=$ $n(-R, y, x)$. This is derived by calculation, using (2.3). Hence the notation $n(R, x)$, in place of $n(R, x, y)$, is unambiguous.

THEOREM 3.6. $N=\langle n(R, x): R \in \Sigma, x \in R \backslash\{1\}\rangle$.

Let $N_{0}=\langle n(R, x) \cdots\rangle$ as above and $H_{0}=N_{0} \cap H$. Then (2.1) through (2.9) are valid with $N, H$ replaced by $N_{0}, H_{0}$ respectively. ( $t$ may be chosen to map into $N_{0}$ ). Hence there is a unique Bruhat decomposition with respect to $N_{0}, H_{0}$, Then $n=u h m v$ where $n \in N, h \in H_{0}$ and $m \in N_{0}$. But $u=v=1$ by (2.1) and (2.3), so $n=h m \in N_{0}$.

LEMMA 3.7. $H=\left\langle n(R, x) n\left(R, x^{\prime}\right): x, x^{\prime} \in R \backslash\{1\}, R \in \Sigma\right\rangle$.

Let $h\left(R, x, x^{\prime}\right)=n(R, x) n\left(R, x^{\prime}\right)$ and $H_{1}=\left\langle h\left(R, x, x^{\prime}\right) \cdots\right\rangle$ as above. The following formulas are established by calculation:

$$
\begin{aligned}
& h\left(R, x, x^{\prime}\right)^{n}=h\left(s(n) R, x^{n}, x^{\prime n}\right) \\
& n(R, x) H_{1} \cdot n\left(R, x^{\prime}\right) H_{1} \subseteq H_{1} \\
& \left(n(S, y) H_{1}\right)^{n(R, x) H_{1}}=n\left(w_{R} S, y^{n(R, x)}\right) H_{1} .
\end{aligned}
$$

Then the mapping $q: n(R, x) H \rightarrow n(R, x) H_{1}$ extends to a homomorphism of $N / H$ onto $N / H_{1}$ which is inverse to the natural homomorphism $N / H_{1} \rightarrow N / H$. Hence $H=H_{1}$. Let $H_{R}=\left\langle h\left(R, x, x^{\prime}\right): x, x^{\prime} \in R \backslash\{1\}\right\rangle$.

THEOREM 3.8. $H=\pi_{1}^{n} H_{R_{i}}$.

Each $H_{R}$ is normal in $H$, and these generate $H$ by (3.7), so $H=\pi_{\Sigma} H_{R}$. For $y=x^{n(R, u)}$ and $y^{\prime}=x^{\prime n(R, u)}$, calculation shows: 


$$
h\left(-R, y, y^{\prime}\right)=h(R, u, x) h\left(R, x^{\prime}, u^{-1}\right),
$$

so $H=\pi_{P} H_{R}$. If $R$ is positive, but not simple, then $w_{i} R<R$ for some $i$, by the properties of $W$. Again by calculation:

$$
h\left(R, x, x^{\prime}\right)=h\left(w_{i} R, x^{n}, x^{\prime n}\right)\left(h\left(R_{i}, y, y^{\prime}\right)\right)^{-1}
$$

where $n=n\left(R_{i}, y^{\prime-1}\right)$. Then the theorem follows by induction on root order.

4. The covering group. In this section, the covering group $\Delta$ of $G$ will be defined, and its' structure worked out. It will itself be a group of Lie type.

For each $R \in \Sigma$, let $R^{*}$ be a set in one-one correspondence with $R$. Disjoint sets $R^{*}, S^{*}$ are to correspond to distinct roots $R, S$. Let $\Delta$ be the group generated by the union of $\Sigma^{*}=\left\{R^{*}: R \in \Sigma\right\}$ subject to all relations of the forms given below:

(A) $x^{*} y^{*}=z^{*}$ for $x^{*}, y^{*}, z^{*} \in R^{*}, R^{*} \in \Sigma^{*}$ and $x y=z$ in $G$.

(B) All relations implied by (2.8). (As Curtis showed in [3], some of these are actually not required to define 4 .)

In general, putting a "*” on something will mean the corresponding thing in 4 . It is clear $p: x^{*} \rightarrow x, x^{*} \in R^{*}, R^{*} \in \Sigma^{*}$ extends to a homomorphism of $\Delta$ onto $G$. A convex subgroup of $G$ is one of the form $\left\langle\left\{R^{*}, S^{*}, \cdots\right\}\right\rangle$ where $\{R, S, \cdots\}$ is convex in $\Sigma, n^{*}\left(R^{*}, x^{*}\right)=$ $x^{*} y^{*} x^{*}$ when $x y x=n(R, x)$ in $N$, and $N^{*}=\left\langle n^{*}\left(R^{*}, x^{*}\right): x^{*} \in R^{*}\right.$, $\left.R^{*} \in \Sigma^{*}\right\rangle . \quad s^{*}: N^{*} \rightarrow W$ is defined by $s^{*}=s p$, and the action of $s^{*}\left(n^{*}\right), n^{*} \in N^{*}$, on $\Sigma^{*}$ by $s^{*}\left(n^{*}\right) R^{*}=\left(s^{*}\left(n^{*}\right) R\right)^{*}$. Define $h^{*}\left(R^{*}, x^{*}, x_{1}^{*}\right)=$ $n^{*}\left(R^{*}, x^{*}\right) n^{*}\left(R^{*}, x_{1}^{*}\right)$ and $H^{*}=\left\langle h^{*}\left(R^{*}, x^{*}, x_{1}^{*}\right): x^{*}, x_{1}^{*} \in R^{*} \backslash\{1\}, R^{*} \in \Sigma^{*}\right\rangle$.

LeMma 4.1. (i) $p$ restricted to a convex subgroup is an isomorphism. (ii) $s^{*}\left(n^{*}\right) R^{*}=\left(R^{*}\right)^{n^{*}}, n^{*} \in N^{*}$ and $R^{*} \in \Sigma^{*}$. (iii) $H^{*}$ is normal in $N^{*}$ and $N^{*} / H^{*} \cong W$. (iv) $\Delta$ has a Bruhat decomposition. $(v)(p, \Delta)$ is a central extension of $G$.

The conditions of Theorem (1.4) of [3] hold for a group of Lie type as defined here. In particular, condition (1.6) of [3] is a slightly more specific version of condition (2.8) of this paper, which is sufficient for the proof to follow. Then (i), (ii), (iii), (iv), and (v) are respectively in (2.5), (2.6), (2.9), (1.4) and (1.4) of [3]. As in [3], the proofs of (i), (ii), and (iii) generally follow those of, respectively, Lemmas $7.1,7.2$ and 7.7 of [11].

THEOREM 4.2. $\Delta$ is a group of Lie type.

By 4.1 (iv), conditions (2.1) through (2.5) hold, except the crystal- 
lographic restriction, which holds since the relation in (2.2) occurs in a convex subgroup. Similarly, conditions (2.8) through (2.11) can be regarded in convex subgroups, while (2.6) through (2.8) follow from definitions here. [In particular, by (2.10) $\Delta$ is a perfect group.]

5. The factor map. It remains to show $(p, \Delta)$ is a universal central extension of $G$. From now on $(q, K)$ is a fixed, arbitrary central extension of $G$. A factor homomorphism $k: \Delta \rightarrow K$ is to be constructed so that $p=q . k$. (From now on, *'s may be dropped from elements in $\Delta$.) $k$ will be constructed by choosing images of the generators of $\Delta$ and verifying conditions $(A)$ and $(B)$.

For the highest root $\Gamma$, of each root conjugacy class under $W$, choose $h_{\Gamma} \in H^{*}$ satisfying (2.10) for the subgroup $\Gamma^{*}$. For each root $R=w \Gamma$, let $h_{R}=h_{\Gamma}^{t *(w)}$. For each $R \in \Sigma$ and each $x \in R^{*}$, choose $k(x) \in q^{-1}(p(x))$ in $K$ so that:

$$
k(x)=\left(k\left(x_{1}\right), k\left(h_{R}\right)\right), \text { when } x=\left(x_{1}, h_{R}\right) \text { in } \Delta .
$$

This is always possible, since each $x \in R^{*}$ appears on the left of exactly one such relation. Choices on the right are arbitrary, being inside a commutator.

Lemma 5.1. If elements of $k\left(R^{*}\right)$ commute in $K$, then $k(x) k(y)=$ $k(x y)$ all $x, y \in R^{*}$.

For unique $x_{1}, y_{1} \in R^{*}, x=\left(x_{1}, h_{R}\right)$ and $y=\left(y_{1}, h_{R}\right)$. Then $x y=$ $\left(x_{1}, h_{R}\right)\left(y_{1}, h_{R}\right)=\left(x_{1} y_{1}, h_{R}\right)$. Also, $k\left(x_{1}\right) k\left(y_{1}\right)=z k\left(x_{1} y_{1}\right)$ for some $z \in \operatorname{ker}(q) \subseteq$ $Z(K)$. Conjugating this relation by $k\left(h_{R}\right)$ and simplifying gives:

$$
k(x) k\left(x_{1}\right) k(y) k\left(y_{1}\right)=z\left(k\left(x_{1} y_{1}\right), k\left(h_{R}\right)\right) k\left(x_{1} y_{1}\right) .
$$

Dividing this relation by the previous one gives the desired relation.

Leмma 5.2. If all commutator relations of length less than $n$, of the form

$$
\left(k\left(x_{R}\right), k\left(x_{S}\right)\right)=\pi k\left(x_{i R+j S}\right)
$$

hold in $K$, so do all such relations of length $n$.

(These are the images in $K$ of relations of type $(B)$ in $\Delta$. Commutativity, as in (5.1), is such a relation of length 1.) Any such relation, of any length, holds modulo an element, say $f\left(x_{R}, x_{S}\right)$, which is in $\operatorname{ker} q \subseteq Z(K)$. Using this, and the induction hypothesis, $f\left(x_{R}, x_{S}\right)$ is "bimultiplicative": $f\left(x_{R}, x_{S}\right) f\left(x_{R}, x_{S}^{\prime}\right)=f\left(x_{R}, x_{S} x_{S}^{\prime}\right)$, and similarly for $x_{R} \cdot$ Choose $h$ by (2.11) and suppose $\left(h, S^{*}\right)=S^{*}$. Conjugate 
$\left(x_{R}, x_{S}\right)=\pi x_{i R+j S}$ by $h$ and apply $k$ to get:

$$
\left(k\left(x_{R}\right), k\left(x_{S}^{h}\right)\right)=f\left(x_{R}, x_{S}^{h}\right) \pi k\left(x_{i R+j S}^{h}\right) .
$$

Suppose $k(x)^{k(h)}=k\left(x^{h}\right)$ for $h \in H^{*}, x \in R^{*}, R \in \Sigma$. Apply $k$ to $\left(x_{R}, x_{S}\right)=$ $\pi x_{i R+j S}$ and then conjugate by $k(h)$ to get:

$$
\left(k\left(x_{R}\right), k\left(x_{S}^{h}\right)\right)=f\left(x_{R}, x_{S}\right) \pi k\left(x_{i R+j S}^{h}\right) .
$$

Comparing, and using multiplicativity, $f\left(x_{R}, x_{S}\right)=f\left(x_{R}, x_{S}^{h}\right), f\left(x_{R},\left(h, x_{S}\right)\right)=$ 1 , so $f$ is identically one on $R^{*}, S^{*}$.

Now to prove $k(x)^{k(h)}=k\left(x^{h}\right)$. For any $R \in \Sigma, x, y \in R^{*}$ such that $x=\left(y, h_{R}\right)$, apply $k$ and conjugate by $k(h)$, so that using $H^{\prime} \subseteq Z(\Delta)$ :

$$
\begin{aligned}
k(x)^{k(h)} & =\left(k\left(y^{h}\right), k\left(\left(h, h_{R}\right)\right) k\left(h_{R}\right)\right)=\left(k\left(y^{h}\right), k\left(h_{R}\right)\right) \\
& =k\left(\left(y^{h}, h_{R}\right)\right)=k\left(x^{h}\right) .
\end{aligned}
$$

REMARKs. This proves $\left(k\left(R^{*}\right), k\left(S^{*}\right)\right)=1$ if $R \neq \pm S$ and $\left(R^{*}, S^{*}\right)=$ 1 in $\Delta$. Also, the proof shows that elements of $k\left(R^{*}\right)$ may be conjugated by elements of $k\left(H^{*}\right)$, by the formula established there.

Lemma 5.3. $k(x)^{k(n)}=k\left(x^{n}\right)$ for $x \in R^{*}, R^{*} \in \Sigma^{*}, n \in N^{*}$.

Let $\Gamma$ be the highest root of a conjugacy class, $x=\left(x_{1}, h_{\Gamma}\right)$ for $x, x_{1} \in \Gamma^{*}$, and $n=t^{*}(w)$. Then:

$$
k(x)^{k(n)}=\left(k\left(x_{1}^{n}\right), k\left(h_{\Gamma}^{n}\right)\right)=k\left(\left(x_{1}^{n}, h_{\Gamma}^{n}\right)\right)=k\left(\left(x_{1}, h_{\Gamma}\right)^{n}\right)=k\left(x^{n}\right) .
$$

The second equality follows from the choice of $k(x)$ 's, since $h_{\Gamma}^{n}=h_{w \Gamma}$ and $x_{1}^{n} \in w \Gamma$. Now suppose for any root $R=w \Gamma ; x \in R^{*}, m=t^{*}(w)$, $n=t^{*}\left(w_{S}\right)$. Conjugate by $k(n)$ :

$$
\begin{aligned}
k(x)^{k(n)} & =k\left(y^{m}\right)^{k(n)}=k(y)^{k(m) k(n)}=k(y)^{k\left(t^{*}\left(w w_{S}\right)\right) k(h) z} \\
& =k\left(y^{t^{*}\left(w w_{S}\right) h}\right)=k\left(x^{n}\right),
\end{aligned}
$$

for $y \in \Gamma^{*}, y^{m}=x$, and $m n=t^{*}\left(w w_{s}\right) h$ for some $h \in H^{*}$. But $H^{*}$ and $t^{*}(W)$ generate $N^{*}$.

6. Commutativity of root subgroups. This section covers the starting point of the induction on length of commutators. By previous remarks, it only remains to show $k\left(R^{*}\right)$ is commutative for $R \in \Sigma . \quad \Lambda$ is the highest root of $\Sigma$.

Lemma $6.1 \quad\left(k\left(\Lambda^{*}\right), k\left(\Lambda^{*}\right)\right)=1$.

By the second part of (2.8), for $x \in \Lambda^{*}$ there exist $r \in R^{*}, s \in S^{*}$ such that 


$$
(r, s)=\pi t_{i j}
$$

where $t_{i j} \in i R^{*}+j S^{*}$ whenever this is a root, and $x=t_{i j}$ for some $i, j$. Hence:

$$
(k(r), k(s))=f(r, s) \pi k(t i j) \cdot k(x) .
$$

Then $k\left(x_{1}\right), x_{1} \in \Lambda^{*}$, commutes with everything in this relation except possible $k(x)$, and hence with it also. Now $k\left(\Lambda^{*}\right)$ is commutative, as is the image of every root subgroup $R^{*}$ conjugate to $\Lambda$, by (5.3).

Since $W$ is transitive on roots of the same length, commutativity of $k\left(R^{*}\right)$ only needs to be demonstrated for one short root. It suffices to consider rank two subgroups.

The short root $R+S$ in $B_{2}$.

$$
\left(k\left(x_{R}\right), k\left(x_{S}\right)\right)=f\left(x_{R}, x_{S}\right) k\left(x_{R+S}\right) k\left(x_{2 R+S}\right)
$$

conjugated by $k\left(x_{R+S}^{\prime}\right)$ :

$$
\begin{aligned}
\left(k\left(x_{R}\right)^{k\left(x_{R+S}^{\prime}\right)}, k\left(x_{S}\right)\right) & =f\left(x_{R}, x_{S}\right) k\left(x_{R+S}\right)^{k\left(x_{R+S}^{\prime}\right)} k\left(x_{2 R+S}\right) \\
& =\left(k\left(x_{2 R+S}^{\prime}\right) k\left(x_{R}\right), k\left(x_{S}\right)\right)=\left(k\left(x_{R}\right), k\left(x_{S}\right)\right)^{k\left(x_{2 R+S}^{\prime}\right)} \\
& =f\left(x_{R}, x_{S}\right) k\left(x_{R+S}\right) k\left(x_{2 R+S}\right) .
\end{aligned}
$$

The short root $2 R+S$ in $G_{2}$.

$$
\left(k\left(x_{R+S}\right), k\left(x_{R}\right)\right)=f\left(x_{R+S}, x_{R}\right) k\left(x_{2 R+S}\right) k\left(x_{3 R+S}\right) k\left(x_{3 R+2 S}\right)
$$

conjugate by $k\left(x_{2 R+S}^{\prime}\right)$ :

$$
\begin{aligned}
\left(k\left(x_{R+S}\right), k\left(x_{R}\right)\right)^{k\left(x_{2 R+S}^{\prime}\right)} & =\left(k\left(x_{R+S}\right), k\left(x_{R}\right)\right)^{k\left(x_{3 R+S}^{\prime}\right) k\left(x_{3 R+2 S}^{\prime}\right)} \\
& =f\left(x_{R+S} x_{R}\right) k\left(x_{2 R+S}\right)^{k\left(x_{2 R+S}^{\prime}\right)} k\left(x_{3 R+S}\right) k\left(x_{3 R+2 S}\right) .
\end{aligned}
$$

These considerations complete the proof of the main theorem. As in $\S 4, \Delta$ is itself a group of Lie type, perfect, and with the same (isomorphic) Weyl group as $G$.

THEOREM 6.2. (Main Theorem). $(p, \Delta)$ is the covering group of $G$.

7. The rank one case. For groups of Lie type of rank one, some modification of the previous definitions and proofs is necessary. Since there is only one positive root, $X$, this now denotes what was called $U$ in (2.1). In (2.2), $W \cong Z_{2}$ now holds, and $\left\{1, w_{0}\right\}$ is a set of coset representatives, so a choice of $t$. $R_{1}=X$ and $X_{w}=X$ or $Y$. In (2.5), $G_{1}$ is just $G=\langle X, Y\rangle$ by (2.6). None of conditions (2.8), (2.9) and (2.11) apply here, and are replaced by:

$(2.8)^{\prime}$ There exist $h, g \in H$ such that $x^{g}=x \cdot x^{h}$ and $x \cdot x^{h} \cdot x^{h h} \neq 1$ for all $x \in X \backslash\{1\}$. $X$ is abelian. 
The definition of $\Delta$ also needs to be revised in the rank one case, with the now inapplicable condition $(B)$ replaced by:

$(B)^{\prime} x^{* n^{*}\left(R^{*}, x_{1}^{*}\right)}=y^{*}$ whenever $R^{*}=X^{*}$ or $Y^{*}, x^{*}, x_{1}^{*} \in R^{*}, y^{*} \in-R^{*}$, and $x^{n\left(R, x_{1}\right)}=y$ in $G$.

Lemma 7.1. (i) $B^{*}=X^{*} H^{*}$ and $N^{*}=\left\langle n^{*}\left(R^{*}, x^{*}\right)\right\rangle$ form a split $(B, N)$ pair of rank one for $\Delta$. (ii) $H^{*}=\left\langle h^{*}\left(R^{*} x^{*} x_{1}^{*}\right)\right\rangle=B^{*} \cap N^{*}$. (iii) $(p, \Delta)$ is a central extension of $G$. (iv) $\Delta=(\Delta, \Delta)$.

(i), (ii) come from Theorem (1.2) of [3]. $\quad\left(H^{*}\right.$ and $N^{*}$ correspond here and there since $\operatorname{ker} p \subseteq H^{*}$.) (iii) also follows from (1.2) of [3], and from [11, Th. 3.1]. (iv) can be shown directly from (2.10).

All the results of $\S 3$ and $\S 4$ remain valid in the rank one case, where applicable and when modified as above. In particular, (4.1) (ii) and condition $(2.8)^{\prime}$ follow from $(B)^{\prime}$ in the definition of $\Delta$.

In $\S 5$ and $\S 6$, Lemmas 5.1 and 5.3 still hold. Hence Lemma 6.1 can be proved using $(2.8)^{\prime}$ as follows: Let $x, y \in X^{*} \backslash\{1\}$ and $(k(x), k(y))=f(x, y) \in Z(K)$. (Here the fact that $X$ is abelian, as given in $(2.8)^{\prime}$, is used.) Then:

$$
\begin{aligned}
f\left(x^{h}, y^{g g}\right) & =f\left(x^{h}, y^{h h} y^{h} y^{h} y\right)=f\left(x^{h}, y^{h h} y^{h}\right) f\left(x^{h}, y^{h} y\right) \\
& =f\left(x, y^{h}\right) f\left(x^{h}, y^{h} y\right)=f\left(x^{h} x, y^{h} y\right)=f\left(x^{g g}, y^{g g}\right) .
\end{aligned}
$$

Hence $(B)^{\prime}$ holds in $K$ by (5.3). Since $(A),(B)^{\prime}$ hold in $K$, Theorem 6.2 now holds for rank one groups of Lie type.

8. Which groups are of Lie type. In this section, references are given to known results which show which Chevalley and twisted groups are of Lie type. With the following exceptions, all these groups are of Lie type:

$$
K \text { has } 2,3 \text { or } 4 \text { elements }
$$

[ $K_{0}$, the fixed field, for twisted groups].

$$
A_{1}(5) \text {. }
$$

$$
\begin{aligned}
& A_{2 n}^{\prime}(K) . \\
& D_{4}^{3}(K) .
\end{aligned}
$$

The first three cases, with a possible partial exception under (i) for twisted groups, are real exceptions. $D_{4}^{3}(K)$ is listed as a possible exception since it was not investigated in detail. The (nonalgebraic) nonnormal forms as defined by M. Suzuki $[12,13]$, of type $B_{2}^{1}\left(2^{2 n+1}\right)$, and by R. Ree $[7,8]$ of types $F_{4}^{\prime}\left(2^{2 n+1}\right)$ and $G_{2}^{1}\left(3^{2 n+1}\right)$ were not considered here. 
For Chevalley groups, all the conditions defining groups of Lie type, except (2.8), can be read off almost directly from conditions (2.1.1) through (2.1.12) of [4]. These conditions are verified there for all Chevalley groups for $|K|>5$. In fact, since (2.1.12) is not needed here, $|K|>4$ suffices. Condition (2.8) may be checked directly by computing the commutator relations as in [1, p. 36].

For the only rank one Chevalley group, $A_{1}(K)$, condition $(2.8)^{\prime}$ is verified in $[4, \S 2.2]$ where $(2.1 .12)$ is discussed, but $|K|>5$ is necessary.

Details for the twisted groups, except types $A_{2 n}^{1}(K), D_{4}^{2}(K)$ and $D_{4}^{3}(K)$ are worked out in $[4, \S 2.5]$. The commutator relations developed there are sufficient for the second part of (2.8) to hold. Similarly, the details for $D_{4}^{2}$ are in $[4, \S 3.1]$. In all these cases, it is the fixed field $K_{0}$ under the automorphism of $K$ which must have more than 4 elements. This might be improved in some cases.

The condition $(2.8)^{\prime}$ doesn't need to be checked in this case, since the only rank one twisted group $A_{2}^{1}(K)$ is one of the groups $A_{2 n}^{1}(K)$. All the groups $A_{2 n}^{1}(K)$ are exceptions, not of Lie type, since the root subgroups are not abelian.

\section{BIBLIOGRAPHY}

1. C. Chevalley, Sur certains groupes simples, Tohoku Math. J. 7 (1955), 14-66.

2. C. W. Curtis, Irreducible representations of finite groups of Lie type, J. Reine Angew. Math. 219 (1965), 180-199.

3. - Central extensions of groups of Lie type, J. Reine Angew. Math. 220 (1965), 174-185.

4. J. Grover, Coverings of groups of Chevalley type, Dissertation, University of California, Los Angeles, 1966.

5. D. Hertzig, On simple algebraic groups, Short communications, Int. Congress Math., Edinburgh, 1958.

6. - Forms of algebraic groups, Proc. Amer. Math. Soc. 12 (1961), 657-660.

7. R. Ree, A family of simple groups associated with the simple Lie algebras of type $\left(F_{4}\right)$, Amer. J. Math. 83 (1961), 401-420

8. - A family of simple groups associated with the simple Lie algebras of type $\left(G_{2}\right)$, Amer. J. Math. 83 (1961), 432-462.

9. R. Steinberg, Variations on a theme of Chevalley, Pacific J. Math. 9 (1959), 875891.

10. - The simplicity of certain groups, Pacific J. Math. 10 (1960), 1039-1041.

11. - Generateurs, relations et revetements des groupes algebriques, Colloq. sur la theorie des groupes algebriques, Bruxelles, 1962.

12. M. Suzuki, A new type of simple groups of finite order, Proc. Nat. Acad, Sci. U.S.A. 46 (1960), 868-870.

13. - On a class of doubly transitive groups, Ann. of Math. 75 (1962), 105-145. 14. J. Tits, Les "formes reeles" des groupes de type $\left(E_{6}\right)$, Seminaire Bourbaki 10 (1957/58), Exposes 152.

15. - Sur la trialité et certains groupes qui s'en deduisent, Paris, Inst. Hautes Etudes Sci. Publ. Math. 2 (1959), 37-84.

16. - Algebraic and abstract simple groups, Ann. Math. 80 (1964), 313-329. 
Received March 26, 1968. The results in this paper are from the author's dissertation. The author wishes to gratefully acknowledge the direction of Professor Robert Steinberg of the University of California, Los Angeles, and the partial support of the National Science Foundation through grants GP-5436 and GP-7913.

UNIVERSITY OF CALIFORNIA, IRVINE 



\section{PACIFIC JOURNAL OF MATHEMATICS}

\section{EDITORS}

H. ROYDEN

Stanford University

Stanford, California

\section{RichaRd PIERCE}

University of Washington Seattle, Washington 98105
J. DugundJI

Department of Mathematics

University of Southern California

Los Angeles, California 90007

BASIL GORDON

University of California

Los Angeles, California 90024

\section{ASSOCIATE EDITORS}
E. F. BECKENBACH
B. H. NeUMANN
F. WOLF
K. YOSHIDA

\section{SUPPORTING INSTITUTIONS}

UNIVERSITY OF BRITISH COLUMBIA CALIFORNIA INSTITUTE OF TECHNOLOGY

UNIVERSITY OF CALIFORNIA

MONTANA STATE UNIVERSITY

UNIVERSITY OF NEVADA

NEW MEXICO STATE UNIVERSITY

OREGON STATE UNIVERSITY

UNIVERSITY OF OREGON

OSAKA UNIVERSITY

UNIVERSITY OF SOUTHERN CALIFORNIA

\author{
STANFORD UNIVERSITY \\ UNIVERSITY OF TOKYO \\ UNIVERSITY OF UTAH \\ WASHINGTON STATE UNIVERSITY \\ UNIVERSITY OF WASHINGTON \\ AMERICAN MATHEMATICAL SOCIETY \\ CHEVRON RESEARCH CORPORATION \\ TRW SYSTEMS \\ NAVAL WEAPONS CENTER
}

The Supporting Institutions listed above contribute to the cost of publication of this Journal, but they are not owners or publishers and have no responsibility for its content or policies.

Mathematical papers intended for publication in the Pacific Journal of Mathematics should be in typed form or offset-reproduced, double spaced with large margins. Underline Greek letters in red, German in green, and script in blue. The first paragraph or two must be capable of being used separately as a synopsis of the entire paper. It should not contain references to the bibliography. Manuscripts, in duplicate if possible, may be sent to any one of the four editors. Please classify according to the scheme of Math. Rev. 36, 1539-1546. All other communications to the editors should be addressed to the managing editor, Richard Arens, University of California, Los Angeles, California, 90024.

50 reprints are provided free for each article; additional copies may be obtained at cost in multiples of 50 .

The Pacific Journal of Mathematics is published monthly. Effective with Volume 16 the price per volume (3 numbers) is $\$ 8.00$; single issues, $\$ 3.00$. Special price for current issues to individual faculty members of supporting institutions and to individual members of the American Mathematical Society: $\$ 4.00$ per volume; single issues $\$ 1.50$. Back numbers are available.

Subscriptions, orders for back numbers, and changes of address should be sent to Pacific Journal of Mathematics, 103 Highland Boulevard, Berkeley, California, 94708.

PUBLISHED BY PACIFIC JOURNAL OF MATHEMATICS, A NON-PROFIT CORPORATION

Printed at Kokusai Bunken Insatsusha (International Academic Printing Co., Ltd.), 7-17. Fujimi 2-chome, Chiyoda-ku, Tokyo, Japan. 


\section{Pacific Journal of Mathematics}

\section{Vol. 30, No. $3 \quad$ November, 1969}

Willard Ellis Baxter, Topological rings with property $(Y) \ldots \ldots \ldots \ldots . \ldots 5$

Sterling K. Berberian, Note on some spectral inequalities of $C . R$.

Putnam ..................................... 573

David Theodore Brown, Galois theory for Banach algebras . ........... 577

Dennis K. Burke and R. A. Stoltenberg, A note on p-spaces and Moore spaces ........................................ 601

Rafael Van Severen Chacon and Stephen Allan McGrath, Estimates of positive contractions....................................... 609

Rene Felix Dennemeyer, Conjugate surfaces for multiple integral problems in the calculus of variations ........................... 621

Edwin O. Elliott, Measures on countable product spaces.............. 639

John Moss Grover, Covering groups of groups of Lie type .............. 645

Charles Lemuel Hagopian, Concerning semi-local-connectedness and cutting in nonlocally connected continua .................. 657

Velmer B. Headley, A monotonicity principle for eigenvalues ........... 663

John Joseph Hutchinson, Intrinsic extensions of rings . . . . . . . . . . . . . 669

Harold H. Johnson, Determination of hyperbolicity by partial

prolongations .................................. 679

Tilla Weinstein, Holomorphic quadratic differentials on surfaces in $E^{3} \ldots 697$

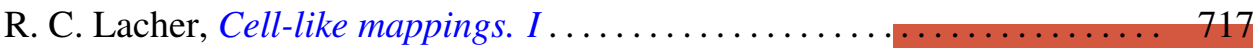

Roger McCann, A classification of centers

Curtis L. Outlaw, Mean value iteration of nonexpansive mappings in a

Banach space...

Allan C. Peterson, Distribution of zeros of solutions of a fourth order

differential equation.

Bhalchandra B. Phadke, Polyhedron inequality and strict convexity .. 765 Jack Wyndall Rogers Jr., On universal tree-like continua .

Edgar Andrews Rutter, Two characterizations of quasi-Frobenius rings

G. Sankaranarayanan and C. Suyambulingom, Some renewal theorems concerning a sequence of correlated random variables...

Joel E. Schneider, A note on the theory of primes........ . .

Richard Peter Stanley, Zero square rings .................

Edward D. Tymchatyn, The 2-cell as a partially ordered space

Craig A. Wood, On general Z.P.I.-rings................ 